BACTERIAL PATHOGENESIS

\section{Weighing up the factors}

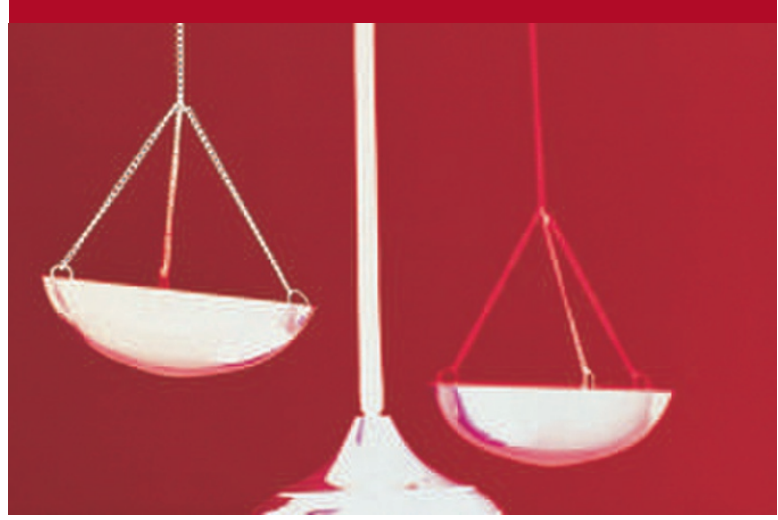

Most microorganisms possess several virulence factors that contribute to overall microbial virulence, the relative capacity of a microorganism to cause damage in a host. Although there are myriad studies of individual virulence factors, so far no methodology had been proposed to study the relative contribution of these virulence factors to the overall virulence phenotype. Now, in the March issue of Infection and Immunity, Erin McClelland, Paul Bernhardt and Arturo Casadevall describe their use of multivariate regression analysis to do just that, using Cryptococcus neoformans and Bacillus anthracis as example organisms.

The main limitation of their method was that, to avoid underpowered results, information is required on a great number of strains that differ in virulence and virulence-factor expression. To overcome this problem, McClelland et al. used a computer program named RandGen to randomly generate data with specified distributions and specific correlations among the variables similar to those observed in actual data sets.

In C. neoformans, the authors analysed the influence of capsule size, melanin production, glucuronoxylomannan release, urease production and growth rate, which are all thought to contribute to virulence, using both experimental and randomly generated data sets. Capsule size was found to be the biggest contributor to virulence, as well as melanin production. For B. anthracis, a microorganism with fewer identified virulence determinants, they demonstrated how the capsule, anthrax toxin and anthralysin virulence factors could be ordered in relative importance using a randomly generated data set.

In this study, McClelland et al. show that multivariate linear regression can be used to determine the contribution of individual virulence factors to overall virulence, as long as an appropriate sample size is used for confident predictions. Such information could be used to select targets for vaccine and drug development.

Annie Tremp
Cryptococcus neoformans http://www.ncbi.nlm.nih.gov/entrez/ query.fcgi? $\mathrm{db}=$ genomeprj\&cmd= Retrieve\&dopt=Overview\&list_ uids $=9581$

Bacillus anthracis

http://www.ncbi.nlm.nih.gov/entrez/ query.fcgi? $\mathrm{db}=$ genomeprj\&cmd= Retrieve\&dopt=Overview\&list_ uids $=12333$ 